\title{
16 EFEITO DA TERAPIA DE REMINISCÊNCIA NA POPULAÇÃO GERIÁTRICA: SCOPING REVIEW
}

\author{
| Andreia Plexa ${ }^{1}$; Maria José Nogueira²; Paulo Seabra ${ }^{3}$; Olga Valentim ${ }^{4} \mid$
}

\section{RESUMO}

CONTEXTO: A Terapia da Reminiscência (TR) tem vindo a ser utilizada com o objetivo de promover o bem-estar, a cognição e o humor. A população geriátrica tem habitualmente associadas patologias como demência e/ou depressão e é frequentemente alvo de TR. Contudo, uma pesquisa preliminar revelou que os dados relativos aos efeitos desta terapia e a forma como esta deve ser implementada, encontram-se dispersos na literatura sendo difícil a sua apreciação. Por conseguinte decidiu-se realizar uma scoping review com a seguinte questão de investigação: "Qual o efeito da terapia da reminiscência na população geriátrica?”.

OBJETIVO(S): Mapear a evidência científica acerca dos efeitos da TR na população geriátrica.

MÉTODOS: Scoping Review orientada pela metodologia proposta pelo Joana Briggs Institute. Entre 1 e 17 de junho de 2018 , foram pesquisados os descritores "reminiscence therapy", "reminiscence" e "life review" nas plataformas EBSCO, B-on, e nas bases de dados PubMed e SciELO. A seleção dos artigos foi realizada de acordo com critérios pré-definidos: idade dos participantes superior a 65 anos, aplicação de TR, artigos gratuitos e disponíveis em texto integral em Português, Espanhol e Inglês.

RESULTADOS: Foram analisados 4 artigos num total de 73. A TR produz efeitos na redução da sintomatologia depressiva e na sobrecarga dos cuidadores, no comportamento, desempenho cognitivo e no bem-estar.

CONCLUSÕES: Existe pouca evidência científica que comprove os efeitos da TR na população geriátrica, e a forma como esta deve ser implementada, pelo que é necessário desenvolver estudos longitudinais com amostras significativas, por forma a melhor compreender o efeito da TR. PALAVRAS-CHAVE: Terapia narrativa; Terapia; Rememoração mental; Saúde mental

\section{RESUMEN}

\section{"Efecto de la terapia de reminiscencia en la población geriátrica: Scoping Review"}

CONTEXTO: La Terapia de la Reminiscencia (TR) ha sido utilizada con el objetivo de promover el bienestar, la cognición y el humor. La población geriátrica generalmente está asociada a la demencia y depresión y está frecuentemente sometida a RT. Una investigación preliminar reveló que los datos sobre los efectos de esta terapia y cómo debe implementarse están dispersos en la literatura y son difíciles de evaluar. Consiguiente, se decidió realizar una scoping review con la siguiente pregunta de einvestigación: "Cuál es el efecto de la terapia de reminiscencia en la población geriátrica?”

OBJETIVO(S): Mapear la evidencia sobre los efectos de la TR en la población geriátrica.

METODOLOGÍA: Scoping Review usando la metodología propuesta por el Instituto Joanna Briggs. Entre el 1 y el 17 de junio de 2018, se usaron los descriptores "reminiscence therapy", "reminiscence" y "life review" en las plataformas EBSCO y B-on, en las bases PubMed y SciELO. La selección de artículos se llevó a cabo de acuerdo con criterios previamente definidos: edad de los participantes superior a 65 años, participantes se sometieron a la TR, y artículos disponibles en texto completo en portugués, español e inglés.

RESULTADOS: Se analizaron 4 artículos de un total de 73. La TR produce efectos en la reducción de la sintomatología depresiva y sobrecarga de los cuidadores, en el comportamiento, cognición y el bienestar.

CONCLUSIONES: Hay poca evidencia científica que demuestre los efectos de la TR en la población geriátrica y de cómo debe ser implementada. Consiguiente es necesario realizar más estudios longitudinales y con muestras significativas para comprender mejor el efecto de la TR.

\section{DESCRIPTORES: Terapia narrativa; Terapia; Recuerdo mental; Salud mental}

\section{ABSTRACT \\ "Effects of reminiscence therapy on the geriatric population: Scoping review"}

BACKGROUND: Reminiscence Therapy (RT) has been used to promote wellbeing, cognition and mood improvement. The geriatric population is usually associated with pathologies such as dementia and/or depression and is often targeted for RT. However, preliminary research has shown that data regarding the effects of this therapy and how it should be implemented are scattered in the literature. Therefore, we decided to carry out a scoping review with the following research question: "What is the effect of reminiscence therapy on the geriatric population?"

AIM: To map the scientific evidence about the effects of the RT in the geriatric population.

METHODS: A Scoping Review guided by the methodology proposed by the Joanna Briggs Institute. From the first of June to the 17th of 2018, we search for the "reminiscence therapy", "reminiscence" and "life review" descriptors in the EBSCO and B-on platforms, at PubMed and SciELO databases. The papers were selected according to predefined criteria such as participants' age of over 65 years, participants submitted to RT, papers with free full text in Portuguese, Spanish and English.

RESULTS: 4 articles were selected and analyzed from a total of 73 . The RT produces effects on the reduction of depressive symptoms and caregivers' overload, behavior, cognition, and well-being.

CONCLUSIONS: There is a lack of evidence to substantiate the effect of the RT on the geriatric population, as well as how it should be applied. Therefore, further longitudinal studies with significant samples are necessary to better understand the effect of RT in the geriatric population.

\section{KEYWORDS: Narrative therapy; Therapy; Mental recall; Men- tal health}

Submetido em 30-12-2018

Aceite em 11-03-2019

1 Estudante de Mestrado em Enfermagem de Saúde Mental e Psiquiátrica na Universidade Católica Portuguesa; Enfermeira no Centro Hospitalar Psiquiátrico de Lisboa, Avenida do Brasil, 53, Lisboa, Portugal, andreiaplexa@chpl.min-saude.pt

2 Enfermeira especialista em Enfermagem de Saúde Mental e Psiquiátrica; Doutora em Enfermagem; Investigadora no Centro de Pesquisa em Tecnologia e Serviços de Saúde (CINTESIS - NursID); Professora Adjunta na Escola Superior de Saúde Atlântica, Barcarena, Portugal, nogueira.mjc@gmail.com

3 Enfermeiro especialista em Enfermagem de Saúde Mental e Psiquiátrica; Doutor em Enfermagem; Investigador na Unidade de Investigação e Desenvolvimento em Enfermagem (UI\&DE); Professor Adjunto na Escola Superior de Enfermagem, Lisboa, Portugal, pauloseabra@esel.pt

4 Enfermeira especialista em Enfermagem de Saúde Mental e Psiquiátrica; Doutora em Enfermagem; Investigadora no Centro de Pesquisa em Tecnologia e Serviços de Saúde (CINTESIS - NursID); Professora Adjunta na Escola Superior de Saúde Atlântica, Barcarena, Portugal, ommvalentim2@gmail.com

Citação: Plexa, A., Nogueira, M. J., Seabra, P., \& Valentim, O. (2020). Efeito da terapia de reminiscência na população geriátrica: Scoping review. Revista Portuguesa de Enfermagem de Saúde Mental (Spe7), 109-114. 
$\mathrm{O}$ artigo "The life review: an interpretation of reminiscence in the aged" publicado em 1963 por Robert Butler, foi pioneiro a contrariar os efeitos nocivos que eram atribuídos ao processo de reminiscência. Segundo o artigo, na época existiam opiniões díspares acerca da natureza e funcionalidade da reminiscência, sendo a mesma desencorajada por se acreditar que poderia conduzir a deterioração mental. Butler (1963) defendia que a reminiscência era um processo mental, natural e universal; de regresso progressivo de experiências passadas à consciência, com enfoque em conflitos não resolvidos, para que pudessem ser integrados promovendo assim, a integridade do ego. $\mathrm{O}$ investigador defendia ainda que este processo era acelerado pela aproximação da morte, conduzindo a uma reorganização interna. Merriam (1993) contraria esta afirmação, referindo que a reminiscência não é um processo universal, precipitado pela aproximação da morte, dado que as pessoas com 100 anos de idade incluídas no estudo realizado não apresentaram maior evidência de reminiscências comparativamente às pessoas de 60 anos. $O$ estudo comprovou ainda que nem todas as pessoas desenvolveram processos de reminiscência, pelo que esta poderá não assumir um carácter universal. Erikson (1959) e Butler (1963) afirmaram que a revisão da vida é uma tarefa a ser realizada em idades avançadas, e esta tem vindo a ser utilizada como intervenção terapêutica, promovendo a autoaceitação e saúde mental das pessoas (Pinquart \& Forstmeier 2011). Os termos reminiscence e life review surgem na literatura como estando relacionados com o mesmo processo, no entanto são conceitos distintos. Butler (1963) identifica reminiscence como um processo de pensar sobre si, sendo transversal e inerente à pessoa, e life review como um processo estruturado e intencional de mobilização de eventos passados com objetivo terapêutico.

Wong \& Watt (1991) diferenciaram seis tipos de reminiscência: integrativa (obter um sentido de autovalor, coerência e reconciliação com o passado), instrumental (contribui para a perceção subjetiva de competência e continuidade), transmissiva (transmissão de heranças culturais e legado pessoal), escapista (tendência a glorificar o passado e depreciar o presente), obsessiva (insucesso na integração de experiências passadas), e narrativa (fornece informação biográfica). No entanto também Webster, Bohlmeijer, \& Westerhof (2010) e Westerhof, Bohlmeijer, \& Webster (2010) realizaram uma distinção entre diferentes tipos de reminiscência.
Esta distinção distingue a reminiscência simples, revisão de vida e terapia de revisão de vida. A reminiscência simples apresenta-se como uma narrativa de memórias autobiográficas não estruturada. A revisão de vida consiste numa intervenção mais estruturada, habitualmente realizada de forma individual, trabalhando memórias positivas e negativas. A terapia de revisão de vida consiste numa abordagem mais terapêutica, focando-se em promover uma visão positiva do passado. Habitualmente integra também técnicas da terapia cognitivo-comportamental, técnica de resolução de problemas ou terapia narrativa.

A aplicação de terapia de reminiscência na população geriátrica é frequente. Os efeitos atribuídos a esta terapia passam pela melhoria da qualidade de vida, bemestar, e desempenho cognitivo. Contudo, os estudos que corroborem estas afirmações são escassos. Assim, esta scoping review é realizada com o intuito de mapear o conhecimento presente na evidência científica acerca do efeito da terapia da reminiscência na população geriátrica, respondendo à questão: "Qual o efeito da terapia da reminiscência na população geriátrica?".

\section{MÉTODOS}

Tendo em conta o método de elaboração da questão de investigação preconizado pelo Joana Briggs Institute (População, Conceito e Contexto (PCC)), foi identificada a população em estudo (população geriátrica), o conceito (efeitos da terapia da reminiscência) e contexto (pessoas sob intervenção em comparação com pessoas não submetidas à intervenção terapia da reminiscência), resultando na questão de investigação: "Quais os efeitos da terapia da reminiscência na população geriátrica?”. Relativamente aos critérios de elegibilidade para seleção dos artigos foram determinados os seguintes: amostra com pessoas com idades superiores a 65 anos, dado que se procura apurar a evidência relativa à intervenção na população geriátrica; aplicação de terapia de reminiscência, excluindo a implementação simultânea com outras terapias; artigos disponíveis em texto integral e gratuito; limite temporal dos últimos dez anos, a fim de se apurar a evidência mais recente; e artigos escritos em português, inglês e espanhol, por serem idiomas compreendidos pelos investigadores. No que diz respeito à tipologia de estudos foram excluídos artigos de opinião, revisão de literatura narrativa, conferências e estudos de caso por não responderem a uma metodologia estruturada. A pesquisa foi efetuada no período de 1 a 17 de junho de 2018 . 
No que concerne à estratégia de pesquisa foi realizada uma primeira pesquisa com os descritores "reminiscence therapy", AND "life review" AND "geriatric" (optamos por manter os conceitos na sua forma original como forma de ampliar a identificação de artigos). Os resultados foram escassos, pelo que se repetiu a pesquisa inserindo o descritor "reminiscence". Contudo o resultado não se alterou significativamente. Assim, alteraram-se os descritores para "reminiscence therapy" AND "reminiscence" AND "life review", obtendo um resultado mais satisfatório. Foram pesquisados os descritores anteriormente referidos, nas plataformas EBSCO (bases de dados CINAHL Complete Full Text e MEDLINE Complete Full Text) e B-on. Na SciELO (Portugal), os descritores utilizados foram "reminiscência" e "saúde". A pesquisa perfez um total de 73 artigos - EBSCO: 26; B-on: 32; PubMed: 11; SciELO PT: 4 (Figura 1). Após a remoção de artigos duplicados permaneceram 31 artigos em análise. A seleção destes artigos foi realizada pela leitura do título, excluindo os artigos sobre temáticas não relacionadas com a TR; pela leitura do resumo, excluindo os artigos sem evidência de metodologia estruturada ou referência à origem da informação; pela leitura integral do texto, seguindo o protocolo PRISMAScR (Tricco et al., 2018). Importa referir que nesta fase final a seleção foi realizada sempre com a validação de pelo menos dois dos investigadores de forma independente. $\mathrm{O}$ total dos artigos apurados foi quatro (Figura 1).

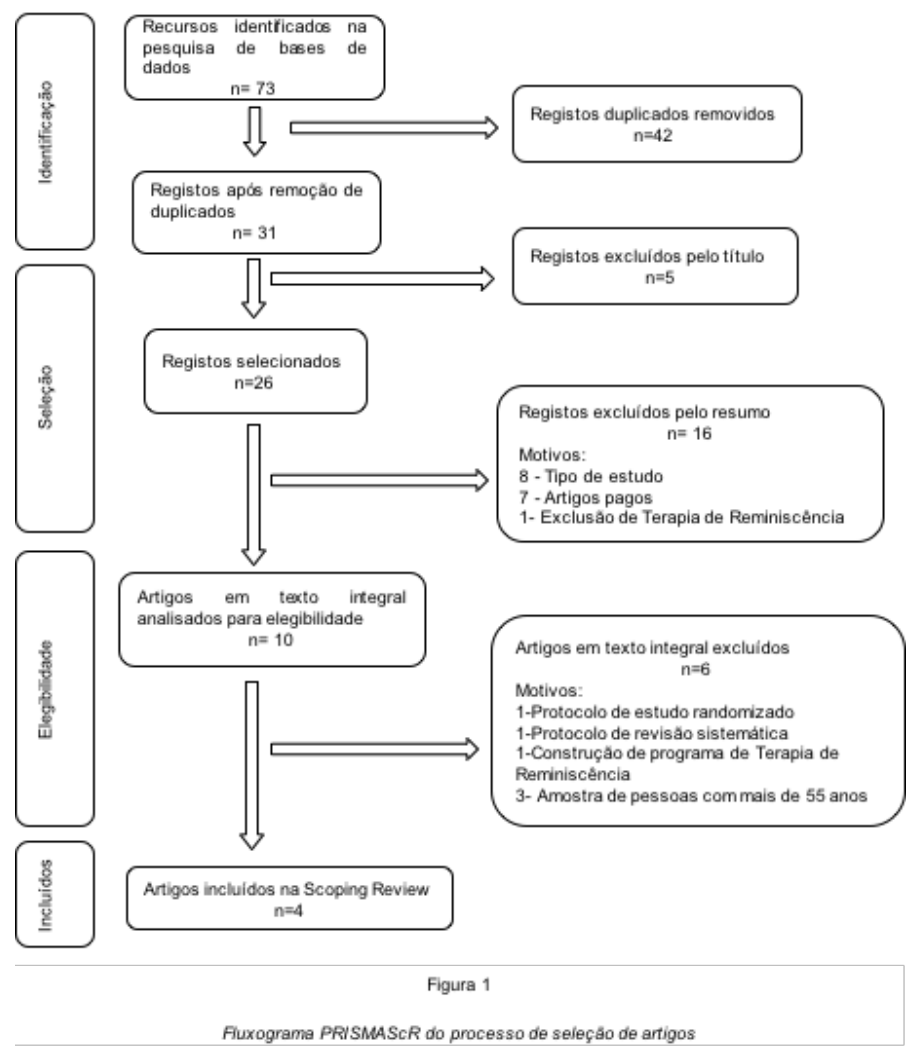

\section{RESULTADOS}

Após a seleção dos artigos, procedeu-se à extração sistematizada da informação relevante para a temática em estudo, que pode ser consultada no Quadro 1.

Quadro 1 - Extração de resultados

\begin{tabular}{|c|c|c|c|c|}
\hline $\begin{array}{l}\text { Autor/ } \\
\text { ano }\end{array}$ & $\begin{array}{l}\text { Desenho } \\
\text { do estudo }\end{array}$ & Objetivo & Amostra & Resultados \\
\hline $\begin{array}{l}\text { Pin- } \\
\text { quart } \\
\& \\
\text { Forst- } \\
\text { meier } \\
2011\end{array}$ & $\begin{array}{l}\text { Meta } \\
\text { análise }\end{array}$ & $\begin{array}{l}\text { Integrar } \\
\text { resultados de } \\
\text { estudos clíni- } \\
\text { cos aleatórios } \\
\text { controlados } \\
\text { (RCT) rela- } \\
\text { tivamente a } \\
\text { determinadas } \\
\text { variáveis (9) } \\
\text { nas fases de } \\
\text { pós-interven- } \\
\text { ção follow up. }\end{array}$ & $128 \mathrm{RCT}$ & $\begin{array}{l}\text { No pós-interven- } \\
\text { ção - melhoria de } \\
\text { todas as variáveis. } \\
\text { No follow-up } 6 \text { das } \\
9 \text { variáveis manti- } \\
\text { veram melhoria. } \\
\text { Terapia de revisão } \\
\text { de vida apresenta } \\
\text { maior eficácia. } \\
\text { Intervenção indi- } \\
\text { vidual não produz } \\
\text { melhores efeitos } \\
\text { que a de grupo. }\end{array}$ \\
\hline $\begin{array}{l}\text { Pre- } \\
\text { schl } \\
\text { et al. } \\
2012\end{array}$ & $\begin{array}{l}\text { Estudo } \\
\text { clínico } \\
\text { aleatório } \\
\text { e contro- } \\
\text { lado }\end{array}$ & $\begin{array}{l}\text { Comparar } \\
\text { TR presencial } \\
\text { com TR com } \\
\text { recurso a uso } \\
\text { do computa- } \\
\text { dor. }\end{array}$ & $\begin{array}{l}36 \text { pes- } \\
\text { soas }\end{array}$ & $\begin{array}{l}\text { Diminuição de } \\
\text { sintomatologia } \\
\text { depressiva. } \\
\text { Aumento de bem- } \\
\text { estar. } \\
\text { Sem alterações } \\
\text { significativas na } \\
\text { qualidade de vida. }\end{array}$ \\
\hline $\begin{array}{l}\text { Lopes, } \\
\text { Afon- } \\
\text { so e } \\
\text { Ri- } \\
\text { beiro } \\
2014\end{array}$ & $\begin{array}{l}\text { Revisão } \\
\text { integra- } \\
\text { tiva da } \\
\text { literatura }\end{array}$ & $\begin{array}{l}\text { Efetuar uma } \\
\text { revisão inte- } \\
\text { grada da lit- } \\
\text { eratura sobre } \\
\text { os principais } \\
\text { efeitos da } \\
\text { reminiscência } \\
\text { em pessoas } \\
\text { com demên- } \\
\text { cia. }\end{array}$ & $\begin{array}{l}28 \text { arti- } \\
\text { gos }\end{array}$ & $\begin{array}{l}\text { Melhoria de } \\
\text { cognição, compor- } \\
\text { tamento, humor, } \\
\text { diminuição de } \\
\text { sintomatologia de- } \\
\text { pressiva, aumento } \\
\text { de bem-estar, } \\
\text { qualidade de vida, } \\
\text { comunicação, } \\
\text { capacidade funcio- } \\
\text { nal e diminuição } \\
\text { sobrecarga dos } \\
\text { cuidadores. }\end{array}$ \\
\hline $\begin{array}{l}\text { Jons- } \\
\text { son } \\
\text { et al. } \\
2016\end{array}$ & $\begin{array}{l}\text { Revisão } \\
\text { sistemáti- } \\
\text { ca }\end{array}$ & $\begin{array}{l}\text { Sintetizar evi- } \\
\text { dência sobre } \\
\text { eficácia, segu- } \\
\text { rança e custo } \\
\text { do tratamento } \\
\text { psicológico } \\
\text { da depressão, } \\
\text { e avaliar a } \\
\text { qualidade } \\
\text { da evidência } \\
\text { encontrada. }\end{array}$ & $22 \mathrm{RCT}$ & $\begin{array}{l}\text { Melhoria de } \\
\text { sintomatologia de- } \\
\text { pressiva, qualidade } \\
\text { de vida, sendo que } \\
\text { apenas um deles } \\
\text { teve efeito positivo } \\
\text { nesta variável. }\end{array}$ \\
\hline
\end{tabular}

A meta análise de Pinquart \& Forstmeier (2011) englobou 128 estudos clínicos aleatórios e controlados dos quais 82 diziam respeito a reminiscência simples, 37 de revisão de vida e 18 de terapia de revisão de vida. Estes 128 estudos dizem respeito a uma amostra total de mais de 4000 participantes, quer nos grupos experimentais, quer nos grupos de controlo. 
Na maioria dos estudos selecionados foi aplicada a terapia em grupo; a distribuição da amostra foi realizada de forma aleatória pelos dois grupos; em média foram praticadas 10 sessões de terapia de reminiscência (médias variaram entre 8 a 9 semanas).

$\mathrm{Na}$ avaliação realizada no momento pós-intervenção, todas as variáveis haviam melhorado significativamente, pela seguinte ordem: integridade do ego, sintomatologia depressiva, propósito de vida, preparação para a morte, mestria, saúde mental, bem-estar, interação social e cognição. No follow up este efeito positivo persistiu em seis das nove variáveis: na sintomatologia depressiva, saúde mental, bem-estar, integridade do ego, cognição e preparação para a morte. Segundo os investigadores este decréscimo também pode estar relacionado com a escassez de estudos (27 em 128) que efetuaram avaliação no follow up. Importa referir que as variáveis analisadas pelos investigadores foram sujeitas a aplicação de instrumentos de avaliação.

Preschl et al. (2012), conduziram um estudo clínico aleatório e controlado com o objetivo de comparar a aplicação da TR com a aplicação da mesma com recurso a computador. A amostra foi recrutada através de anúncios em diversos locais como jornais, lojas, e farmácias; seguindo-se uma seleção dos participantes de acordo com critérios previamente estabelecidos. Os sujeitos com défice cognitivo, depressão grave, ideação suicida, diminuição acentuada da acuidade visual e auditiva, e a receber psicoterapia foram excluídos. A alocação aos grupos foi feita de forma aleatória. A intervenção teve a duração de 6 semanas. A avaliação das variáveis foi realizada no pré e pós-intervenção e no follow up (três meses). Verificou-se que a sintomatologia depressiva diminuiu de forma significativa, inclusive ao momento do follow up. A mobilização de reminiscência de tipo obsessivo diminuiu, e verificouse ainda aumento do bem-estar sentido pelos participantes. Os investigadores especularam acerca da possibilidade dos participantes terem continuado a usar estratégias aprendidas durante a intervenção, e assim o desenvolvimento de melhores mecanismos de coping, o que justificaria os resultados positivos obtidos no follow up. A hipótese colocada de que a reminiscência integrativa poderia aumentar falhou, não se observando alterações significativas. A qualidade de vida sentida pelos participantes também não sofreu alterações significativas. Como limitações foram apontadas a amostra reduzida, e o facto de $30 \%$ da mesma ter sido submetida previamente a psicoterapia.
Lopes, Afonso e Ribeiro (2014) realizaram uma revisão integrativa da literatura com o objetivo de identificar os principais efeitos da reminiscência em pessoas com demência. Dos 28 estudos incluídos na revisão, 11 eram estudos experimentais, 6 quase-experimentais e 11 não experimentais. Os domínios cognição, comportamento, depressão/humor, comunicação, qualidade de vida, bem-estar e atividades de vida diárias foram avaliados com instrumentos de avaliação prédefinidos. A variável cognição apresentou melhoria significativa em 5 dos 18 estudos, comportamento melhorado verificou-se em 11 dos 16, depressão diminuída em 5 de 8 estudos, humor melhorado em 3 dos 6 , comunicação com efeito positivo em 4 de 5 , a qualidade de vida promovida em 3 de 5, bem-estar aumentado em 4 de 5 , e finalmente as atividades de vida diárias beneficiaram em 1 dos 5 estudos encontrados. Relativamente ao impacto da reminiscência nos cuidadores um estudo apresenta uma diminuição na sua sobrecarga. $\mathrm{O}$ stresse e a saúde em geral não apresentam melhorias significativas nos estudos consultados pelos investigadores. Como limitações são apontadas as amostras reduzidas dos estudos, critérios de inclusão pouco definidos e falta de desenhos longitudinais.

Jonsson et al. (2016) realizaram uma revisão sistemática com o objetivo de sintetizar evidência sobre eficácia, segurança e custo do tratamento psicológico da depressão. Dos 22 estudos clínicos aleatórios e controlados apenas 3 diziam respeito a TR. Estes apresentavam amostras pequenas. $\mathrm{O}$ número de sessões aplicadas foi heterogéneo, bem como as características das intervenções. Os 3 estudos apresentaram efeitos positivos na sintomatologia depressiva, 2 estudos incluíram também a qualidade de vida, sendo que apenas 1 deles teve efeito positivo nesta variável. Os investigadores consideraram como limitações a amostra reduzida nos estudos e possíveis enviesamentos nos estudos que comprometeram a sua qualidade.

\section{DISCUSSÃO}

Os resultados encontrados nos artigos demonstram claramente que a terapia da reminiscência é uma intervenção que promove o bem-estar, a qualidade de vida e a melhoria da saúde mental das pessoas. Pinquart \& Forstmeier (2011) afirmam que a terapia da reminiscência produz ligeiras a moderadas melhorias na sintomatologia depressiva e noutras variáveis referentes à saúde mental. 
Esta meta análise apresentou efeitos moderados na integridade do ego, o que apoia a sugestão de Butler (1963), que afirmava que as intervenções baseadas em reminiscência são úteis no desenvolvimento de uma atitude de autoaceitação. No entanto, os investigadores lamentam existirem poucos estudos com diferentes formas de reminiscência. Ainda assim, nos resultados apresentados, a reminiscência simples não promoveu alterações significativas quando comparada com a reminiscência enquanto processo não-terapêutico. Ainda de acordo com Pinquart \& Forstmeier (2011) a terapia da reminiscência pode ser utilizada também para promoção de bem-estar, e não apenas quando se pretende intervir em sintomatologia depressiva. No entanto, estes autores ressalvam as suas limitações na prevenção, apresentando alterações pouco significativas quando se fala em promoção de bem-estar e prevenção de patologias. Este resultado corrobora o estudo realizado por Ercan-Sahin \& Emiroglu (2018) que estudou o impacto da terapia de reminiscência na qualidade de vida de idosos institucionalizados sem patologia psiquiátrica e defeito cognitivo (critério de exclusão). Os investigadores concluíram a terapia de reminiscência não conduziu a melhoria da qualidade de vida dos participantes. Como possível justificação dos achados os autores apontam questões culturais, dado que a sociedade em estudo prefere a permanência no domicílio à institucionalização.

A revisão de literatura de Lopes et al. (2014) refere que a terapia da reminiscência tem um impacto positivo nos participantes dos estudos analisados que sofriam de demência e seus cuidadores. Os mesmos investigadores produziram um estudo quase-experimental (2016) que atesta melhorias a nível da cognição, diminuição da sintomatologia depressiva e ansiedade no grupo experimental, comparativamente ao grupo de controlo. Estas variáveis vão de encontro às variáveis avaliadas pela meta-análise de Pinquart \& Forstmeier (2011).

Preschl et al. (2012) conduziram um estudo pioneiro na aplicação da terapia da reminiscência com recurso a um programa informático, apresentando alterações muito significativas no decréscimo da sintomatologia depressiva. Contudo referem não poder generalizar os resultados do seu estudo, atendendo ao tamanho da amostra, ao risco de enviesamento pela homogeneidade dos participantes, e pela ausência de tratamento aplicada ao grupo controlo.
Jonsson et al. (2016) referem que as intervenções de domínio psicoterapêutico, que incluem a terapia da reminiscência, apresentam-se como eficazes a integrarem o tratamento da depressão nas pessoas idosas, e apontam como limitações encontradas nos estudos selecionados a falta de coerência conceptual, utilizandose termos como "reminiscence" e "life review" como "quase" sinónimos; escassez de estudos relativos a diferentes tipos de reminiscência; amostras pouco significativas; e escassez de estudos longitudinais. Lopes et al. (2014) apontaram ainda como limitação o facto de os instrumentos de avaliação por vezes utilizados não serem sensíveis a patologias como a demência.

Relativamente às limitações desta scoping review, os autores consideram que pode ser apontada como limitação a não utilização de descritores DeCS ou MeSH, o que conduziu possivelmente a um número reduzido de artigos, no entanto, tal deveu-se ao facto do termo "reminiscence" não estar incluído no browser MeSH mas ser utilizado na literatura como termo de referência. Também, o facto de não ter sido usada qualquer combinação de Thesaurus, os idiomas, e os tipos de estudos selecionados podem ter ainda limitado o acesso a artigos com relevante evidência científica.

\section{CONCLUSÕES}

Com esta scoping review foi possível identificar alguns dos efeitos que a terapia de reminiscência produz nas pessoas idosas, apresentando-se com elevado potencial terapêutico, principalmente na população idosa com demência ou síndrome depressivo. Contudo, é necessário a realização de mais estudos, metodologicamente consistentes, com amostras significativas, principalmente de tipo longitudinal, por forma a compreender melhor os efeitos desta terapia, além do seu efeito imediato. A concordância em termos de clareza concetual também é um fator a estudar, bem como, a necessidade de se investigar modelos estruturados de intervenção no âmbito da terapia de reminiscência.

A evidência proveniente do único estudo primário encontrado revela a efetividade desta técnica na diminuição da sintomatologia depressiva e aumento de bem-estar, indicando que esta revisão traz implicações para a prática clínica e para a investigação. Também o facto de se terem encontrado poucos artigos em 2017 e 2018, que cumpram os critérios pré-definidos, pode justificar a necessidade de mais estudos, pelo que esta revisão pode ser um contributo importante como ponto de partida. 


\section{IMPLICAÇÕES PARA A PRÁTICA CLÍNICA}

A utilização da terapia de reminiscência, enquanto intervenção psicoterapêutica com a população idosa, apresenta indícios de produzir efeitos positivos, na sintomatologia depressiva, no bem-estar, na capacidade cognitiva, no autoconceito, pelo que a sua implementação deve ser mantida, a par do seu estudo, e partilha de achados. Acresce nesta reflexão para a prática clínica a evidência do seu efeito na diminuição da sobrecarga dos cuidadores de pessoas com demência. Portanto, considera-se que esta tipologia de intervenção, que carece à partida de uma clarificação conceptual, deve ser implementada por diferentes grupos profissionais que trabalham com pessoas idosas e adultas. Há evidência de benefício, existem programas estruturados que podem ser implementados ou replicados.

\section{REFERÊNCIAS BIBLIOGRÁFICAS}

Butler, R. (1963). The life review: an interpretation of reminiscence in the aged. Psychiatry. 26(1), 65-76. Doi: $10.1080 / 00332747.1963 .11023339$

Ercan-Sahin, N., \& Emiroglu, O. N. (2018). Quality of life of nursing home residents before and after reminiscence therapy. Educational Gerontology, 44, 99107. Doi: $10.1080 / 03601277.2017 .1415115$

Erikson, E. (1959). Identity and the life cycle: Selected papers. Psychological Issues, 29(1),105-126. Doi: 10.1080/21674086.1960.11926165

Joanna Briggs Institute. (2015). Joanna Briggs Institute Reviewers Manual 2015. South Australia: Autor. Disponível em: https://nursing.lsuhsc.edu/JBI/docs/ ReviewersManuals/Scoping-.pdf

Jonsson, U., Bertilsson, G., Allard, P., Gyllensvärd, H., Söderlund, A., Tham, A., \& Andersson, G. (2016). Psychological treatment of depression in people aged 65 years and over: a systematic review of efficacy, safety, and cost-effectiveness. Plos One, 11(8), e0160859. Doi: $10.1371 /$ journal.pone.0160859

Lopes, T., Afonso, R., e Ribeiro, O. (2014). Impacto de intervenções de reminiscência em idosos com demência: revisão de literatura. Psicologia, Saúde \& Doenças, 15(3), 597-611. Doi: 10.15309/14psd150304
Lopes, T., Afonso, R., e Ribeiro, O. (2016). A quasi-experimental study of a reminiscence program focused on autobiographical memory in institutionalized older adults with cognitive impairment. Archives of Gerontology and Geriatrics, 66, 183-192. Doi: 10.1016/j. archger.2016.05.007

Merriam, S. (1993). Butler's life review: How universal is it? International journal of aging and human development, 37, 163-175. Doi: 10.2190/RTKY-8VUDHBEG-12L8

Pinquart, M., \& Forstmeier, S. (2011). Effects of reminiscence interventions on psychosocial outcomes: a meta-analysis. Aging \& Mental Health, 16(5), 541558. Doi: 10.1080/13607863.2011.651434

Preschl, B., Maercker, A., Birgit, W., Forstmeier, S., Baños, R., Alcañiz, M., Castilla, D., \& Botella, C. (2012). Life-review therapy with computer supplements for depression in the elderly: a randomized controlled trial. Aging \& Mental Health, 16(8), 964-974. Doi: $10.1080 / 13607863.2012 .702726$

Tricco, A. C., Lillie, E., Zarin, W., O’Brien, K. K., Colquhoun, H., Levac, D., Moher, D., Peters, M.D.J., Horsley, T., Hempel, S., Akl, E.A., Chang, C., McGowan, J., Stewart, L., Hartling, L., Aldcroft, A., Wilson, M.G., Garritty, C., Lewin, S., Godfrey, C. M., Macdonald, M.T., Langlois, E.V., Soares-Weiser, K., Moriarty, J., Clifford, T., Tunçalp, O., \& Straus, S.E. (2018). PRISMA Extension for Scoping Reviews (PRISMAScR): Checklist and Explanation. Annals of Internal Medicine,169(7), 467-473. Doi: 10.7326/ M18-0850

Webster, J., Bohlmeijer, E., \& Westerhof, G. (2010). Mapping the future of reminiscence: a conceptual guide for research and practice. Research on Aging, 32, 527-564. Doi: 10.1177/0164027510364122

Westerhof, G., Bohlmeijer, E., \& Webster, J. (2010). Reminiscence and mental health: a review of recent progress in theory, research and interventions. Ageing \& Society, 30, 697-721. Doi: 10.1017/ S0144686X09990328

Wong, P., \& Watt, L. (1991). What types of reminiscence are associated with successful aging? Psychology and Aging, 6, 272-279. Doi:10.1037/0882-7974.6.2.272 